\title{
Collapse of optical binding under secondary irradiation
}

\author{
David L. Andrews* and Justo Rodríguez \\ Nanostructures and Photomolecular Systems, School of Chemical Sciences, University of East Anglia, \\ Norwich NR4 7TJ, United Kingdom \\ *Corresponding author: david.andrews@physics.org
}

Received May 28, 2008; revised July 8, 2008; accepted July 11, 2008; posted July 15, 2008 (Doc. ID 96579); published August 7, 2008

\begin{abstract}
When an optically trapped and bound micrometer-sized chain of particles is subjected to a suitably oriented secondary laser beam above a threshold level of intensity, the structure will collapse. The effect arises from modifications to the interparticle energy landscapes owing to the superposition of optically induced potentials. From the theory it also emerges that, for particles separated by near-field distances, optically induced assemblies may be continuously transformed between linear, spherical, and lamellar forms. The results show scope for the optical fabrication of moldable nanoscale structures. (C) 2008 Optical Society of America OCIS codes: $270.5580,020.7010,120.4880,190.5890,220.4241$.
\end{abstract}

Optical binding is a subject of considerable current interest [1-6]. First identified by Thirunamachandran in theoretical work based on quantum electrodynamics (QED) [7], and subsequently demonstrated experimentally by Burns et al. [8], it is a phenomenon now widely recognized to offer unprecedented opportunities for the nanoscale manipulation of matter. Already, a variety of optically induced configurations and interparticle forces have been reported for particle pairs and multiparticle arrays $[3-5,9,10]$. To develop the general theory, some recent investigations focusing on near-field behavior have employed a semiclassical approximation [11-14], while other extensive calculations have reverted to the use of QED $[15,16]$. The phenomenon is still not yet fully understood, and at this juncture it is important to entertain experiments that will allow comparisons to be made between experimental and theoretical results-to consolidate an understanding of the underlying mechanisms, as well as to achieve new techniques for optical manipulation.

A recurrent motif in studies of optical binding is the use of counterpropagating laser beams. This is a device that ensures the beam radiation pressure cancels out, and any optically induced potential is the only significant force over nanoscale distances where the irradiance is effectively constant [7]. Here, we consider one such laser setup, supplemented by the addition of a secondary laser throughput designed to modify the total field the particles experience. With reference to a simple particle pair, Fig. 1 illustrates the wave-vector and polarization-vector geometry, also defining parameters for establishing their orientation relative to the interparticle displacement vector. Two counterpropagating beams (primary beams $\mathbf{k}_{1}$ and $\mathbf{k}_{2}$, the standard configuration) are located on the $x$ axes, each having the same polarization in the $y$ direction. These beams are intersected orthogonally by a secondary field, $\mathbf{k}_{3}$, propagating in the $y$ direction with $x$-axis polarization; the latter, on mirror reflection, propagates back as $\mathbf{k}_{4}$ with a polarization ro- tated by $\pi / 2$ (through the employment of an optical element not shown in the diagram). The net field the particles experience, in the region where the lasers cross, can then be adapted by controlling the relative irradiance of the primary and secondary beams. As will be shown, the resulting optical perturbation of the particles determines the way they are arranged.

Previous quantum electrodynamical studies of the optically induced interparticle potential have revealed intricate features in the energy landscapes, suggesting that a variety of optically induced multiparticle structures, forces, and torques can be induced by a single plane wave [1]. When the four plane waves in the setting of Fig. 1 are taken into account, an expression of the following form, modified from the normal plane-wave result, emerges for the interparticle potential energy:

$$
\begin{aligned}
\Delta E= & \left(\frac{k^{3} \alpha_{0}^{A} \alpha_{0}^{B}}{16 \pi^{2} c \epsilon_{0}^{2}}\right)\left(\left[I_{s} D_{1}+2 I_{p} C_{1}\right] \frac{\cos k R}{k R}\right. \\
& \left.+\left[I_{s} D_{3}+2 I_{p} C_{3}\right]\left(\frac{\cos k R}{k^{3} R^{3}}+\frac{\sin k R}{k^{2} R^{2}}\right)\right) .
\end{aligned}
$$

Here, $I_{p}$ and $I_{s}$ are the irradiances of the primary and secondary counterpropagating beams, respectively; $\alpha_{0}^{A}=\alpha_{0}^{A}(-k, k)$ and $\alpha_{0}^{B}=\alpha_{0}^{B}(-k, k)$ are the frequencydependent dynamic polarizabilities of the two particles $A$ and $B ; C_{n}=\left(1-n \cos ^{2} \phi\right) \cos (k R \sin \phi \cos \zeta)$ and $D_{n}=\left(2-n \sin ^{2} \phi\right) \cos (k R \cos \phi)$; and $k$ is $2 \pi$ times the wavenumber of the incident light. The two angles that feature in $C_{n}$ and $D_{n}$ are $\phi$, the angle between the displacement vector $\mathbf{R}$ and the $y$ axis, and $\zeta$, the angle between the $\mathbf{R}$ projection on the $z-x$ plane and the $x$ axis.

In deriving Eq. (1), each pair of counterpropagating beams is assumed to have equal and opposite Poynting vectors, though in practice their wavelengths can be marginally offset to preclude interference phenomena associated with holographic optical trapping. 


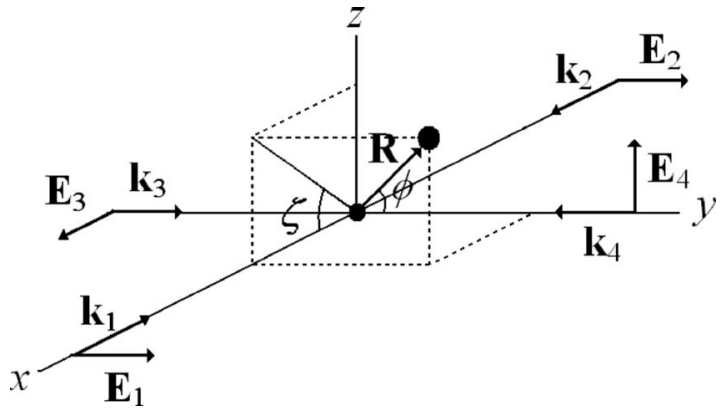

Fig. 1. Pair of particles irradiated by two counterpropagating beams designated primary, $\left(\mathbf{E}_{1}, \mathbf{k}_{1}\right)$ and $\left(\mathbf{E}_{2}, \mathbf{k}_{2}\right)$ (standard optical binding), and secondary beams, $\left(\mathbf{E}_{3}, \mathbf{k}_{3}\right)$ reflected with transverse polarization as $\left(\mathbf{E}_{4}, \mathbf{k}_{4}\right)$. The angle subtended by the interparticle displacement vector $\mathbf{R}$ on the $y$ axis is $\phi$, and the angle between the $\mathbf{R}$ projection on the $z-x$ plane and the $x$ axis is $\zeta$.

Let us now consider some of the interesting features of the variations in interparticle potential that may be observed by controlling the irradiances $I_{p}$ and $I_{s}$. In the near field, (for particle separations $k R \ll 1$ ), and when $I_{s} \neq 2 I_{p}$, the dominant term in the expression for the pair energy may be written as

$$
\Delta E=\left[\frac{\left(2 I_{p}-I_{s}\right) \alpha_{0}^{A} \alpha_{0}^{B}}{16 \pi^{2} c \epsilon_{0}^{2}}\right] \frac{\left(1-3 \cos ^{2} \phi\right)}{R^{3}} .
$$

With $I_{s}>2 I_{p}$, such that the secondary beam exerts the principal optical trapping force directed along the $y$ axis, the first term in brackets in Eq. (2) is negative, and for any given particle separation a potential energy minimum will occur at $\phi= \pm \pi / 2$. This indicates that, under such conditions, an assembly of many particles can group in a lamellar form on the $x-z$ plane of Fig. 1. Conversely, when $2 I_{p}>I_{s}$, the term in brackets is positive, and the potential energy minimum for a fixed particle separation is at $\phi=0$ or $\pm \pi$, in this case, a multiparticle system will line up along the $y$ axis.

The special case where $2 I_{p}=I_{s}$ merits careful attention, as the approximate limiting result given by Eq. (2) is no longer applicable. Moreover, in applying the full result (1), the $R$ dependence of the $C_{n}$ and $D_{n}$ parameters needs to be taken into account. Developing Eq. (1) for the short range $k R \ll 1$, use of l'Hôpital's rule reveals that the term in $\left[I_{s} D_{3}+2 I_{p} C_{3}\right]$ generates a leading contribution that, like the preceding $\left[I_{s} D_{1}\right.$ $\left.+2 I_{p} C_{1}\right]$ term, runs with $R^{-1}$. Thus, the interparticle potential is proportional to the inverse power of the particles separation; moreover it is always attractive. The result indicates that a system of particles, subjected to these conditions, will agglomerate into a spherical form. (A similar effect can be engineered in Bose-Einstein condensates, see [17].)

We can now appreciate the behavior that can be expected, in the configuration shown in Fig. 1, when particles initially trapped in a conventional counterpropagating beam setup are subjected to secondary beams of increasing intensity. At first, with particles separated by small distances, the Casmir-Polder potential might be considered to be of considerable im- portance owing to its inverse sixth power distance dependence, enabling a group of trapped particles to form a compact structure. However, as we have shown previously [18], the laser irradiances employed in optical trapping are usually high enough for optical binding forces to dominate, and the particles line up along the polarization direction, here the $y$ axis. By smoothly increasing the irradiance of the secondary laser (and consequently also the reflected field), the particle system is passively modified to a spherical form when the irradiance of the secondary field becomes equal to the irradiance of the counterpropagating beams. On further increase of the secondary beam irradiance, the sphere is continuously modified to a lamellar form transverse to the secondary field propagation direction. The initial structure can be recovered by reducing the irradiance of the secondary field.

When the particle separations are larger, in the order of magnitude of the laser wavelength, different arrays may be formed. At first, when the secondary beam is off, $I_{s}=0$, the particles experience only standard optical binding; therefore, they may line up along the propagation direction of the primary beams (longitudinal optical binding) with particles separated by $k R \cong 3$ [1]. When the secondary beam, $I_{s}$, is brought into play, and its irradiance increased, the potential energy dependence as a function of particle separations is modified as shown in Fig. 2; the minimum mainly responsible for the primary formation of particle chains disappears-the structure is no longer stable. A detailed view of the effect on the potential surface, of increasing the irradiance of the

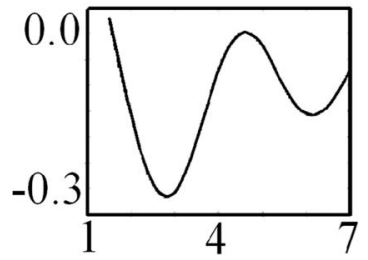

(a)

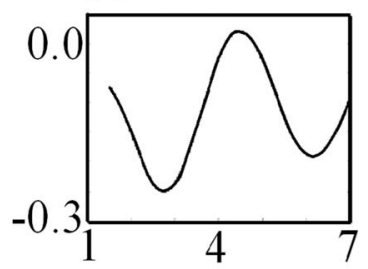

(b)

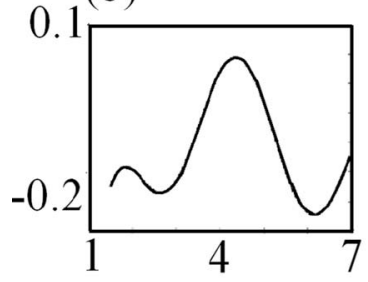

(c)

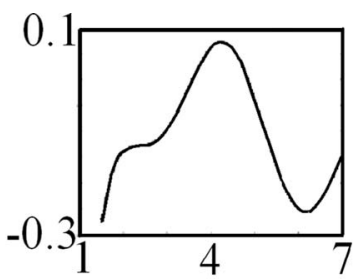

(d)

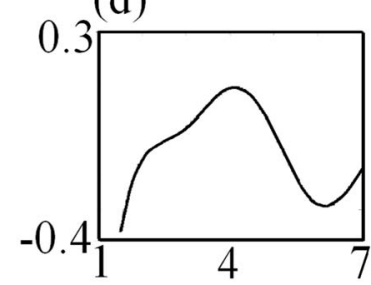

(e)

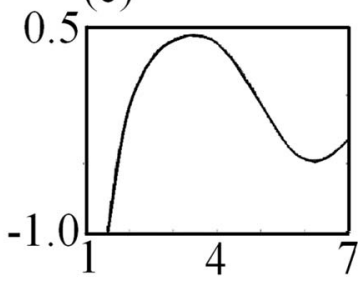

(f)
Fig. 2. Interparticle potential energy as a function of $k R$ for $\zeta=0$ and $\phi=\pi / 2$, when $I_{s}$ is (a) 0.0 , (b) $0.4 I_{p}$, (c) $0.8 I_{p}$, (d) $1.2 I_{p}$, (e) $1.6 I_{p}$, and (f) $4.0 I_{p}$. The units of energy are $\left[I_{p} k^{3} \alpha_{0}^{A} \alpha_{0}^{B} /\left(16 \pi^{2} c \varepsilon_{0}^{2}\right)\right]$. 
secondary beam, is shown in the mpg file supplied, which illustrates the evolution of the potential surface for particles on the $x-y$ plane of Fig. 1 . The interaction energy responsible for the formation of the linear arrangement of particles (the beginning of the video, $I_{s}=0$, for $\phi=\pi / 2$ ) is captured in Fig. 2(a), and successive parts of the figure correspond to stills. The most striking feature is the disappearance of the most prominent potential energy minimum originally located at $k R \sim 3$ (with $\phi=\pi / 2$ ), which then renders a particle chain a nonstable configuration. The effect occurs at an intensity threshold $I_{s} \cong 1.2 I_{p}$, as illustrated in Fig. 2(d). Physically, this signifies that a chain of particles that might originally extend in space over a distance of the order of micrometers collapses to a very much smaller structure, where the Casimir-Polder forces or short-range optical binding will dominate.

In conclusion, we have demonstrated that a variety of effects can be produced by introducing a secondary pair of counterpropagating laser beams, transverse to a conventional optical binding setup. According to their intensity, the secondary beams can significantly modify the potential energy landscape to which trapped particles are subjected, and the local minima that correspond to stable configurations become strongly dependent on the relative irradiances of the primary and secondary beams. In particular, when an optically trapped and bound chain of micrometersized particles is subjected to a secondary beam above a threshold level of intensity, the structure will collapse. Moreover, for particles separated by nearfield distances, optically induced assemblies prove continuously transformable between linear, spherical, and lamellar forms.

\section{References}

1. L. C. Dávila Romero, J. Rodríguez, and D. L. Andrews, Opt. Commun. 281, 865 (2008).

2. R. G. Crisp and D. L. Andrews, Proc. SPIE 6483, 648304 (2007).

3. F. Chen, G. L. Klimchitskaya, V. M. Mostepanenko, and U. Mohideen, Opt. Express 15, 4823 (2007).

4. N. K. Metzger, R. F. Marchington, M. Mazilu, R. L. Smith, K. Dholakia, and E. M. Wright, Phys. Rev. Lett. 98, 068102 (2007).

5. M. Guillon, O. Moine, and B. Stout, Phys. Rev. Lett. 96, 143902 (2007).

6. S. Ahlawat, R. Dasgupta, and P. K. Gupta, Proc. SPIE 6535, 65350W (2007).

7. T. Thirunamachandran, Mol. Phys. 40, 393 (1980).

8. M. M. Burns, J.-M. Fournier, and J. A. Golovchenko, Phys. Rev. Lett. 63, 1233 (1989).

9. S. K. Mohanty, J. T. Andrews, and P. K. Gupta, Opt. Express 12, 2746 (2004).

10. C. D. Mellor, T. A. Fennerty, and C. D. Bain, Opt. Express 14, 10079 (2006).

11. F. Depasse and J.-M. Vigoureux, J. Phys. D 27, 914 (1994).

12. M. Nieto-Vesperinas, P. C. Chaumet, and A. Rahmani, Philos. Trans. R. Soc. London, Ser. A 362, 719 (2004).

13. D. Maystre and P. Vincent, J. Opt. A 8, 1059 (2006).

14. T. M. Grzegorczyk, B. A. Kemp, and J. A. Kong, Phys. Rev. Lett. 96, 113903 (2006).

15. D. L. Andrews, R. G. Crisp, and D. S. Bradshaw, J. Phys. B 39, S637 (2006).

16. D. S. Bradshaw and D. L. Andrews, Phys. Rev. A 72, 033816 (2005).

17. D. O’Dell, S. Giovanazzi, G. Kurizki, and V. M. Akulin, Phys. Rev. Lett. 84, 5687 (2000).

18. J. Rodriguez, L. C. Dávila Romero, and D. L. Andrews, J. Nanophotonics 1, 019503 (2007). 